\title{
Spin transfer torque enhancement in dual spin valves in the ballistic regime
}

\author{
P. Yan, ${ }^{1}$ Z. Z. Sun, ${ }^{2}$ and X. R. Wang ${ }^{1}$ \\ ${ }^{1}$ Physics Department, The Hong Kong University of Science and Technology, Clear Water Bay, Hong Kong SAR, China \\ ${ }^{2}$ Institute for Theoretical Physics, University of Regensburg, D-93040 Regensburg, Germany
}

(Received 21 June 2010; published 17 May 2011)

\begin{abstract}
The spin transfer torque in an all-metal dual spin valve, in which two antiparallel-aligned pinned ferromagnetic layers are on the two sides of a free ferromagnetic layer with two thin normal metal spacers in between, is studied in the ballistic regime. It is argued that, similar to the results in the diffusive regime, the spin transfer torque is dramatically enhanced in comparison to that in a conventional spin valve in the ballistic regime. Within the Slonczewski approach, an analytical expression of the torque on the free magnetic layer is obtained, which may serve as a theoretical model for the micromagnetic simulation of the spin dynamics in a dual spin valve. Depending on the orientation of the free layer and the degree of electron polarization, the spin transfer torque enhancement could be tenfold. The general cases when transmission and reflection probabilities of the free layer are different from zero or one are also numerically calculated.
\end{abstract}

DOI: 10.1103/PhysRevB.83.174430

PACS number(s): 72.25.Ba, 75.60.Jk, 85.70.Ay, 85.75.-d

\section{INTRODUCTION}

Current-induced magnetization reversal of magnetic multilayers has attracted much research interest due to its rich physics and potential applications in spintronic devices. ${ }^{1-8} \mathrm{~A}$ spin valve, consisting of two ferromagnetic layers and one normal metal spacer in between, is one of such multilayer structures. In a spin valve, one of the magnetic layers, acting as a spin polarizer, is thick so conducting electrons are polarized after passing through it. The polarized conducting electrons transfer their spin angular momentums to local magnetization of the thinner free magnetic layer, resulting in the spin transfer torque (STT) effect first proposed by Slonczewski ${ }^{9}$ and Berger. ${ }^{10}$ Although the STT has many advantages over a magnetic field in manipulating the magnetization state, ${ }^{11,12}$ a large current density is needed to achieve a technologically useful magnetization switching speed ${ }^{13,14}$ but the associated Joule heating could affect device performance. Therefore, the current density reduction becomes a challenging issue from the spintronics application viewpoint. ${ }^{15}$

Many efforts have been devoted to the issue, including using optimized time-dependent current pulse, ${ }^{16}$ pure spin current, ${ }^{17}$ and thermal activation. ${ }^{18,19}$ One direct approach is to increase the magnitude of the STT under a given current through unique geometry design. In 2003, Berger ${ }^{20}$ proposed a novel magnetic multilayer architecture called the dual spin valve (DSV), where the free magnetic layer is sandwiched between two thicker pinned magnetic layers with opposite magnetizations. It is predicted that the STT applied on the free magnetic layer should be much larger than that in a traditional spin valve for a given current in the diffusive regime. ${ }^{20}$ The argument is that spins accumulate at both interfaces of the free layer and STT is proportional to spin accumulations..$^{20}$ However, it is not clear whether this STT enhancement can occur in a DSV in the ballistic regime. Moreover, the analytical formalism for STT in a DSV is still an open problem. ${ }^{21,22}$ These are the focuses of the present paper. A quantum description of the STT in metallic magnetic DSV, valid when both the mean free path and the spin-flip relaxation length are larger than the thickness of the spacers, is presented. Averaged STT (over electron phases) is obtained analytically within the Slonczewski semiclassical approach $^{9}$ when all magnetic layers are perfect polarizers. It is found that STT in a DSV, depending on the orientation of the free layer and the degree of electron polarization, can be enhanced by a factor of tens in comparison with that in a spin valve. The general cases of arbitrary transmission and reflection coefficients of the free layer are also numerically calculated.

This paper is organized as follows: In Sec. II, a physical picture of STT origin in both a spin valve and a DSV is presented. Section III gives the theoretical model and formulation of the electron transport through a DSV. The results, including the analytical expression of the STT on the free layer within the Slonczewski approach, and discussions are given in Sec. IV. Section V is the summary.

\section{PHYSICAL PICTURE}

It is useful to present first a physical picture about STT origin in a spin valve and, in particular, its enhancement in a DSV. Consider a spin valve, which is schematically shown in Fig. 1(a), consisting of a pinned ferromagnetic layer $\mathrm{FM}_{A}$ and a free ferromagnetic layer $\mathrm{FM}_{B}$ separated by a normal metal spacer NM. The magnetizations of $\mathrm{FM}_{A}$ and $\mathrm{FM}_{B}$ are represented by unit vectors, $\mathbf{m}_{A}$ and $\mathbf{m}_{B}$, and their saturated magnetizations. To demonstrate the principle, we assume in this section that all ferromagnetic layers are perfect spin filters, such that spins aligned parallel with the layer magnetization can completely transmit through the layer while antiparallel spins are totally reflected. In this ideal case, a closed analytical solution of the STT can be obtained, which is shown later.

Consider electrons flowing from the left to the right in Fig. 1(a). The right-moving electrons are initially polarized along the $\mathbf{m}_{A}$ direction after passing through $\mathrm{FM}_{A}$. They retain their spin polarization when they impinge on $\mathrm{FM}_{B}$ as long as the spacer thickness is much shorter than the spin-diffusion length, which is usually the case in nanoscale spin valves. ${ }^{23}$ Because the polarization direction of $\mathrm{FM}_{B}$ is noncollinear with that of $\mathrm{FM}_{A}$, an electron polarized along $\mathbf{m}_{A}$ is the superposition of two states along $\mathbf{m}_{B}$ and $-\mathbf{m}_{B}$, so 

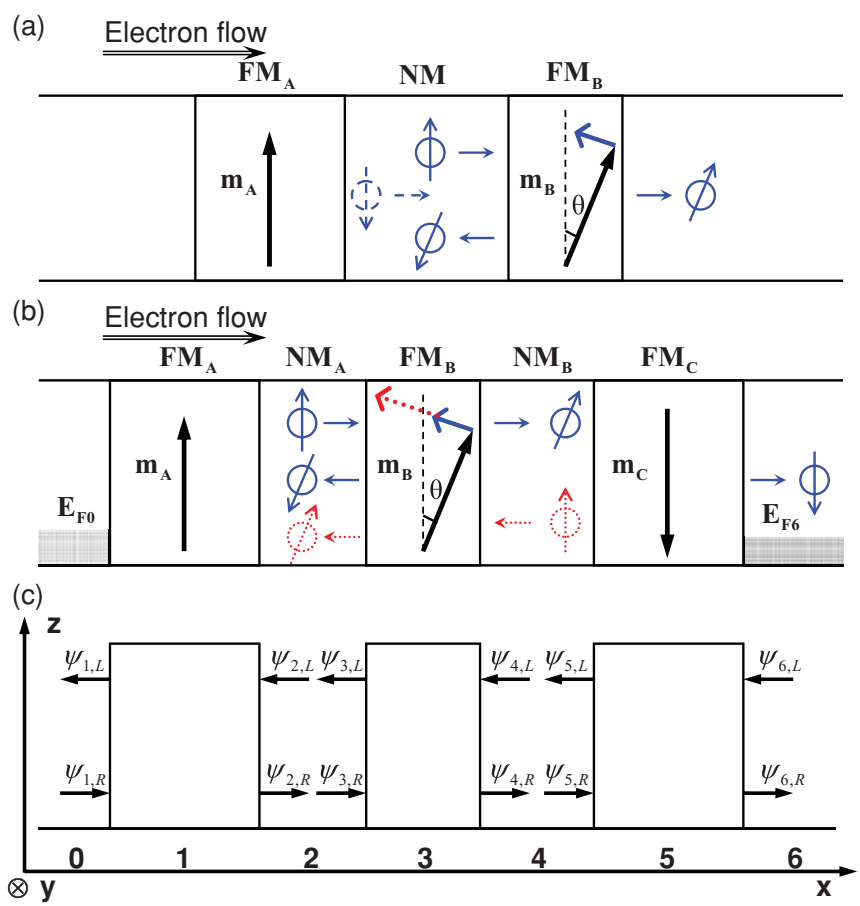

FIG. 1. (Color online) (a) Illustration of STT generated in a spin valve. The circles with arrows illustrate the electron flow with spin-polarization direction. (b) Schematic explanation of STT enhancement in a DSV; $V_{b}=E_{F 0}-E_{F 6}$ is the applied bias. $\mathrm{FM}_{A, B, C}$ are ferromagnetic layers and $\mathrm{NM}_{A, B}$ are normal metal layers. (c) Wave functions at the interfaces of regions 0-6 in a DSV and the coordinates orientation.

the component along $\mathbf{m}_{B}$ can transmit through $\mathrm{FM}_{B}$ while that along $-\mathbf{m}_{B}$ is totally reflected since $\mathrm{FM}_{B}$ is a perfect polarizer. Thus, there is a net angular momentum transfer, perpendicular to $\mathbf{m}_{B}$, from impinged electrons to $\mathrm{FM}_{B}$, resulting in a torque on $\mathrm{FM}_{B}$ to align its magnetization toward $\mathbf{m}_{A}$, as shown by the blue arrow in Fig. 1(a). This is the origin of STT in the spin valve. It should be pointed out that the subsequent multiple reflections of electrons within the NM spacer by the two FM-NM interfaces reduce the STT, since the reflected electrons [dashed symbols in Fig. 1(a)] from $\mathrm{FM}_{B}$ to $\mathrm{FM}_{A}$ and back to $\mathrm{FM}_{B}$ exert a torque along $-\mathbf{m}_{A}$. However, the net torque is not zero since the reflected electrons have a smaller flux than that of the original injected electrons.

Let us now examine the spin transfer in a DSV shown in Fig. 1(b). On the top of a traditional spin valve [Fig. 1(a)], an additional pinned ferromagnetic layer $\mathrm{FM}_{C}$ with an antiparallel-aligned magnetization to $\mathrm{FM}_{A}$ (i.e., $\mathbf{m}_{C}=-\mathbf{m}_{A}$ ) is added so that the free layer $\mathrm{FM}_{B}$ is now sandwiched between $\mathrm{FM}_{A}$ and $\mathrm{FM}_{C}$, separated by two normal metal spacers $\mathrm{NM}_{A}$ and $\mathrm{NM}_{B}$, respectively. Similar to the case of a spin valve, right-moving electrons transmitting through $\mathrm{FM}_{A}$ exert STT on $\mathrm{FM}_{B}$ to align $\mathbf{m}_{B}$ with $\mathbf{m}_{A}$, as shown by the blue arrow in Fig. 1(b). After the electrons transmit through $\mathrm{FM}_{B}$, most of them are reflected by $\mathrm{FM}_{C}$ and then impinge again on $\mathrm{FM}_{B}$, as shown by red dashed symbols in Fig. 1(b). Thus, they exert another STT on $\mathrm{FM}_{B}$ along the $-\mathbf{m}_{C}=\mathbf{m}_{A}$ direction, resulting in the STT enhancement. Multiple reflections in region 2 [Fig. 1(c)] tend to reduce the torque but do not cancel it totally. In the following sections we verify this physical picture through a quantum mechanics calculation.

\section{MODEL AND FORMULATION}

Single charge and spin transport theory in metallic magnetic multilayers is well developed and approaches vary, from classical Valet-Fert theory in the diffusive regime, ${ }^{24}$ to matrix Boltzmann equation formalism, ${ }^{25}$ to the KorringaKohn-Rostoker multiple scattering scheme, ${ }^{26}$ to quantummechanical treatments. ${ }^{9,27-32}$ In the present paper, we adopt a quantum-mechanical description called the scattering matrix method $^{30-32}$ that is valid for ballistic transmission. STT requires the interaction between itinerant electrons and local magnetic moments. In metallic magnetic multilayers, the spin torque can be related to the divergence of a spin current, ${ }^{33}$ which makes the concept and calculation simple ${ }^{27}$ and is used in Sec. IV B. We assume that interfaces are flat and clean and that spin-flip processes are negligible if the spin-flip relaxation length is larger than the spacer thickness ${ }^{23}$ so that momentum $\mathbf{k}$ is a good quantum number in each layer under sufficiently low bias $V_{b}$ on the DSV. Wave functions at the interface of layers labeled by $n=1, \ldots, 6$ as shown in Fig. 1(c) can be written as a two-component spinor multiplied by spatial plane wave,

$$
\psi_{n, \alpha}(x)=\left(\begin{array}{c}
\chi_{n, \alpha, \uparrow} \\
\chi_{n, \alpha, \downarrow}
\end{array}\right) e^{i x\left(k_{x}\right)_{n, \alpha}},
$$

where $\alpha$ denotes propagation directions $[\alpha=L$ for leftward and $\alpha=R$ for rightward with $\left(k_{x}\right)_{n, L}=-k_{x}$ and $\left(k_{x}\right)_{n, R}=$ $k_{x}$ ], $k_{x}$ is the $x$ component of the wave vector, $\chi_{n, \alpha, \uparrow(\downarrow)}$ is the spin-up (spin-down) probability amplitude, the $z$ axis is along $\mathbf{m}_{A}$, and $\mathbf{m}_{B}$ is specified by a polar angle $\theta$ and an azimuthal angle $\phi$.

Incoming and outgoing spinor states in each region are connected to each other by a scattering matrix. ${ }^{30-32}$ In the region $n(=1,2,3,4,5), \psi_{n, L}$ and $\psi_{n+1, R}$ are outgoing spinors while $\psi_{n, R}$ and $\psi_{n+1, L}$ are incoming spinors. They relate to each other by a scattering matrix $\hat{S}_{n}, 34$

$$
\left(\begin{array}{c}
\psi_{n, L} \\
\psi_{n+1, R}
\end{array}\right)=\hat{S}_{n}\left(\begin{array}{c}
\psi_{n, R} \\
\psi_{n+1, L}
\end{array}\right) .
$$

Note that $\psi_{6, L}=0$ since electrons flow from the left reservoir to the right one. The matrix $\hat{S}_{n}$ is a $4 \times 4$ matrix and can be expressed by transmission and reflection coefficients of each scattering region,

$$
\hat{S}_{n}=\left(\begin{array}{cc}
\hat{r}_{n} & \hat{t}_{n} \\
\hat{t}_{n} & \hat{r}_{n}
\end{array}\right),
$$

where $\hat{t}_{n}\left(\hat{r}_{n}\right)$ with $n=1,2,3,4,5$ are $2 \times 2$ transmission (reflection) matrices for layers $\mathrm{FM}_{A}, \mathrm{NM}_{A}, \mathrm{FM}_{B}, \mathrm{NM}_{B}$, and $\mathrm{FM}_{C}$, respectively.

We first treat the pinned ferromagnetic layers $\mathrm{FM}_{A}$ and $\mathrm{FM}_{C}$ as perfect polarizers. Hence, $\hat{t}_{1}, \hat{r}_{1}, \hat{t}_{5}$, and $\hat{r}_{5}$ take the following forms:

$$
\hat{t}_{1}=\hat{r}_{5}=\left(\begin{array}{ll}
1 & 0 \\
0 & 0
\end{array}\right), \quad \hat{r}_{1}=\hat{t}_{5}=\left(\begin{array}{ll}
0 & 0 \\
0 & 1
\end{array}\right) .
$$


Since there are no scatterings in normal metal layers $\mathrm{NM}_{A}$ and $\mathrm{NM}_{B}$ and the propagation of electrons in these layers accumulate dynamical phases, we have

$$
\hat{t}_{2}=\exp \left(i \varphi_{A}\right) \hat{I}, \quad \hat{t}_{4}=\exp \left(i \varphi_{B}\right) \hat{I}, \quad \hat{r}_{2}=\hat{r}_{4}=0,
$$

where $\varphi_{A}$ and $\varphi_{B}$ are the corresponding phase shifts and $\hat{I}$ is the $2 \times 2$ unit matrix. The scattering matrix for $\mathrm{FM}_{B}$ can be expressed by the angles $\theta$ and $\phi$ as

$$
\begin{aligned}
& \hat{t}_{3}=\hat{R}(\theta, \phi) \mathbf{t} \hat{R}^{-1}(\theta, \phi), \\
& \hat{r}_{3}=\hat{R}(\theta, \phi) \mathbf{r} \hat{R}^{-1}(\theta, \phi),
\end{aligned}
$$

where $\hat{R}(\theta, \phi)$ is the rotation that brings $\hat{z}$ into $\mathbf{m}_{B}$ :

$$
\hat{R}=e^{-\frac{i \phi}{2} \sigma_{z}} e^{-\frac{i \theta}{2} \sigma_{y}}=\left(\begin{array}{cc}
e^{-\frac{i \phi}{2}} \cos \frac{\theta}{2} & -e^{-\frac{i \phi}{2}} \sin \frac{\theta}{2} \\
e^{\frac{i \phi}{2}} \sin \frac{\theta}{2} & e^{\frac{i \phi}{2}} \cos \frac{\theta}{2}
\end{array}\right) .
$$

The rotation transformation connects the transmission and reflection matrices between two quantization axes, i.e., $\mathbf{m}_{B}$ and the $z$ axis. Matrices $\mathbf{t}$ and $\mathbf{r}$ are the transmission and reflection matrices when $\mathbf{m}_{B}$ is chosen as the quantization axis:

$$
\mathbf{t}=\left(\begin{array}{cc}
t_{u} & 0 \\
0 & t_{d}
\end{array}\right), \quad \mathbf{r}=\left(\begin{array}{cc}
r_{u} & 0 \\
0 & r_{d}
\end{array}\right),
$$

where $t_{u}, t_{d}, r_{u}$, and $r_{d}$ are transmission and reflection parameters. Subscripts $u$ and $d$ stand for spin-up (majority) and spin-down (minority), respectively. These parameters are complex numbers in general. If $\mathrm{FM}_{B}$ is a perfect polarizer, $t_{u}=1, t_{d}=0$ and $r_{u}=0, r_{d}=1$.

To find the STT on free layer $\mathrm{FM}_{B}$, we need to obtain the scattering states at the two interfaces of $\mathrm{FM}_{B}$. The spindependent scattering wave functions can be expressed in terms of incoming wave $\psi_{1, R}$, such as $\psi_{n, \alpha}=\hat{P}_{n, \alpha} \psi_{1, R}(n=3,4,6)$, where matrices $\hat{P}_{n, \alpha}$ are given by

$$
\begin{gathered}
\hat{P}_{3, R}=\left[\left(\hat{I}-\hat{t}_{2}^{2} \hat{r}_{1} \hat{r}_{3}\right)-\hat{t}_{2}^{2} \hat{t}_{4}^{2} \hat{r}_{1} \hat{t}_{3} \hat{r}_{5}\left(\hat{I}-\hat{t}_{4}^{2} \hat{r}_{3} \hat{r}_{5}\right)^{-1} \hat{t}_{3}\right]^{-1} \hat{t}_{2} \hat{t}_{1}, \\
\hat{P}_{3, L}=\hat{r}_{3} \hat{P}_{3, R}+\hat{t}_{4} \hat{t}_{3} \hat{r}_{5} \hat{Q} \\
\hat{P}_{4, R}=\hat{t}_{4}^{-1} \hat{Q}, \hat{P}_{4, L}=\hat{t}_{4} \hat{r}_{5} \hat{Q} \\
\hat{P}_{6, R}=\hat{t}_{5} \hat{Q}
\end{gathered}
$$

with

$$
\begin{aligned}
\hat{Q}= & {\left[\left(\hat{I}-\hat{t}_{4}^{2} \hat{r}_{3} \hat{r}_{5}\right)-\hat{t}_{2}^{2} \hat{t}_{4}^{2} \hat{t}_{3}\left(\hat{I}-\hat{t}_{2}^{2} \hat{r}_{1} \hat{r}_{3}\right)^{-1} \hat{r}_{1} \hat{t}_{3} \hat{r}_{5}\right]^{-1} } \\
& \times \hat{t}_{3}\left(\hat{I}-\hat{t}_{2}^{2} \hat{r}_{1} \hat{r}_{3}\right)^{-1} \hat{t}_{2} \hat{t}_{4} \hat{t}_{1} .
\end{aligned}
$$

The explicit forms of the above matrices are given in the Appendix.

\section{RESULTS AND DISCUSSIONS}

\section{A. Charge current}

An applied bias $V_{b}$ shown in Fig. 1(b) generates a charge current $J_{e}$ and a spatially dependent spin current $\mathbf{J}_{s}$ through the device. At zero temperature, the charge current reads

$$
J_{e}=\int d E \sum_{\mathbf{q}} j_{e}(\mathbf{q})
$$

with charge current density

$$
j_{e}(\mathbf{q})=e \frac{\hbar k_{x}}{m} \psi_{6, R}^{\dagger} \psi_{6, R},
$$

where $\mathbf{q}$ is the transverse wave vector lying in the $y-z$ plane with energy $E, k_{x}^{2}+\mathbf{q}^{2}=2 m E / \hbar^{2}$, and $e, m$, and $\hbar$ are the electron charge, electron mass, and the Planck constant, respectively. The charge current density can also be written as

$$
j_{e}=e \frac{\hbar k_{x}}{m} T_{e}\left(z_{1}, z_{2}\right),
$$

with transmission coefficient

$$
T_{e}\left(z_{1}, z_{2}\right)=\frac{\left|Q^{\downarrow \uparrow}\left(z_{1}, z_{2}\right)\right|^{2}}{\left|D\left(z_{1}, z_{2}\right)\right|^{2}},
$$

where the explicit forms of parameters $Q^{\downarrow \uparrow}$ and $D$ can be found in the Appendix. In the case that electrons propagate ballistically through $\mathrm{NM}_{A}$ and $\mathrm{NM}_{B}$, the phase shifts in normal metals are given by $\varphi_{A}=k_{x} l_{A}$ and $\varphi_{B}=k_{x} l_{B}$ where $l_{A}$ and $l_{B}$ are the widths of $\mathrm{NM}_{A}$ and $\mathrm{NM}_{B}$, respectively. For sufficiently thick (much bigger than electron Fermi wavelength but still smaller than the spin diffusion length) NM layers, $\varphi_{A}$ and $\varphi_{B}$ vary rapidly from state to state. Thus, when one sums up contributions from different electronic states (different $k_{x}$ ), it is justifiable to assume $\varphi_{A}$ and $\varphi_{B}$ to be random, ${ }^{31}$ and $z_{1}=\exp \left(i 2 \varphi_{A}\right)$ and $z_{2}=\exp \left(i 2 \varphi_{B}\right)$ are equally distributed on the unit circle of the complex plane. ${ }^{30,31}$ However, one should note that $z_{1}$ and $z_{2}$ are not independent under the ballistic assumption since $z_{2}=\left(z_{1}\right)^{p}$ with $p=l_{B} / l_{A}$. The average transmission coefficient is then

$$
\begin{aligned}
\left\langle T_{e}\right\rangle & =\frac{1}{\pi} \int_{0}^{\pi} T_{e}\left(\varphi_{A}, p \varphi_{A}\right) d \varphi_{A} \\
& =\frac{1}{2 \pi i} \oint_{C} \frac{T_{e}\left(z_{1},\left(z_{1}\right)^{p}\right)}{z_{1}} d z_{1},
\end{aligned}
$$

where $C$ is the contour $\left|z_{1}\right|=1$. The contour integral for $p=$ 1 , corresponding to the symmetric DSV configuration, ${ }^{22,35}$ is

$$
\left\langle T_{e}\right\rangle=\sum_{l=1}^{s} \operatorname{Res}\left(\frac{T_{e}\left(z_{1}\right)}{z_{1}} ; z_{1, l}\right),
$$

where $z_{1, l}$ is the $l$ th pole of function $T_{e}\left(z_{1}\right) / z_{1}$ and $s$ is the total number of poles inside the unit circle. In the case when $\mathrm{FM}_{B}$ is perfect, i.e., $t_{u}=1$ and $t_{d}=0\left(r_{u}=0\right.$ and $\left.r_{d}=1\right)$, the function $T_{e}\left(z_{1}\right) / z_{1}$ has only a second-order pole $z_{1}=0$ inside the unit circle. Thus, we can get the average transmission

$$
\left\langle T_{e}\right\rangle\left(t_{u}=1, t_{d}=0\right)=\frac{\sin ^{2} \theta}{2} .
$$

Figure 2 is the average transmission coefficient versus angle $\theta$ for (a) perfect polarizers, (b) the (001) interface of $\mathrm{Au}-\mathrm{Fe},(\mathrm{c})$ the (001) interface of $\mathrm{Cu}-\mathrm{Co},(\mathrm{d})$ the (110) interface of $\mathrm{Cu}-\mathrm{Co}$, and (e) the (111) interface of $\mathrm{Cu}-\mathrm{Co}$. The model parameters for Figs. 2(b)-2(e) are obtained from Ref. 36, where they were extracted from the first-principles calculations. All transmission and reflection amplitudes are assumed to be real. We find that the average total transmission approaches zero as $\theta$ goes to 0 or $\pi$ even if $t_{d}$ is finite for the minority electrons, which is different from the result (Fig. 3 in Ref. 30) in a traditional spin valve. The reasons are as follows. For $\theta=0$, all electrons are polarized along $\mathbf{m}_{A}$ after passing 


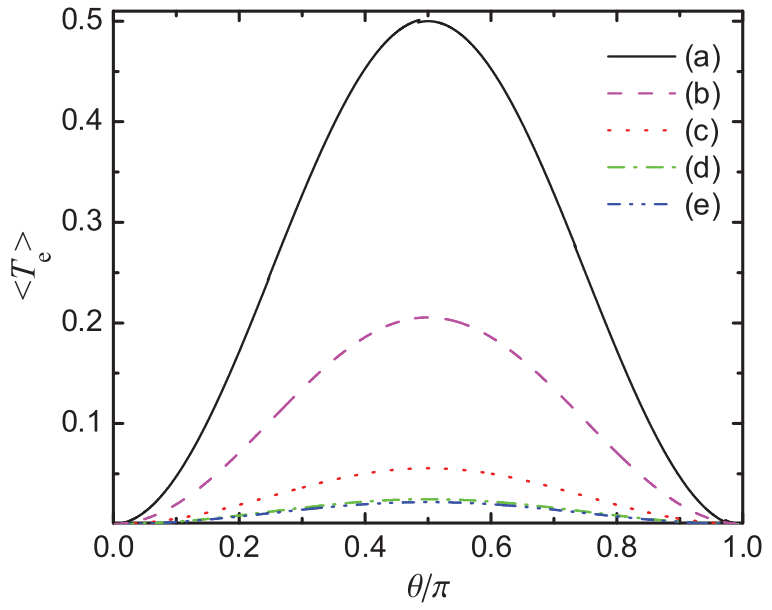

FIG. 2. (Color online) Average transmission coefficient vs angle $\theta$ for (a) $t_{u}=1$ and $t_{d}=0$, (b) $\left|t_{u}\right|^{2}=0.84$ and $\left|t_{d}\right|^{2}=0.17$, (c) $\left|t_{u}\right|^{2}=0.79$ and $\left|t_{d}\right|^{2}=0.49$, (d) $\left|t_{u}\right|^{2}=0.66$ and $\left|t_{d}\right|^{2}=0.44$, and (e) $\left|t_{u}\right|^{2}=0.73$ and $\left|t_{d}\right|^{2}=0.54$.

through $\mathrm{FM}_{B}$. They are totally reflected by $\mathrm{FM}_{C}$ because $\mathbf{m}_{C}=-\mathbf{m}_{A}$, leading to zero electric current. On the other hand, for $\theta=\pi$, all electrons that transmit $\mathrm{FM}_{A}$ are completely reflected by $\mathrm{FM}_{B}$ if it is a perfect polarizer because electron spins are antiparallel to $\mathbf{m}_{B}$, while electrons passing through $\mathrm{FM}_{B}$ maintain polarization along $\mathbf{m}_{A}$ in the case of $t_{d} \neq 0$ and they are totally reflected by $\mathrm{FM}_{C}$ because $\mathbf{m}_{C}=-\mathbf{m}_{A}$. Hence, the average transmission in a DSV vanishes at $\theta=0$ or $\pi$.

The total charge current flowing through the DSV can be obtained by summing Eq. (13) over the transverse momentum q. To find an analytical expression, we adopt the semiclassical Slonczewski approach. ${ }^{9}$ Within the Stoner description of magnetism and letting $\Delta$ be the exchange energy of two spin bands of $\mathrm{FM}_{B}$, one can define two Fermi wave vectors $K_{+}$and $K_{-}$for majorities and minorities,

$$
K_{+}=\sqrt{2 m E / \hbar^{2}}, \quad K_{-}=\sqrt{2 m(E-\Delta) / \hbar^{2}} .
$$

For a ferromagnetic metal, we assume that the Fermi energy lies above the exchange potential, and electrons in NMs are ideally matched with the majority electrons in FM, i.e., $k=K_{+}$. The possible momentum states that contribute to the current can be divided into three ranges ${ }^{9,30}$ :

(a) $0 \leqslant q<K_{-}$. Electrons of both spins in these states contribute to charge current $J_{a}$ :

$$
\begin{aligned}
J_{a} & =2 e \frac{\hbar}{(2 \pi)^{2} m} \int_{0}^{K_{-}} \sqrt{K_{+}^{2}-q^{2}} q d q \\
& =\frac{2}{3} e \frac{\hbar}{(2 \pi)^{2} m}\left[\left(K_{+}^{2}\right)^{3 / 2}-\left(K_{+}^{2}-K_{-}^{2}\right)^{3 / 2}\right] .
\end{aligned}
$$

(b) $K_{-} \leqslant q<K_{+}$. Only majority spin electrons contribute to current $J_{b}$ :

$$
\begin{aligned}
J_{b} & =e \frac{\hbar}{(2 \pi)^{2} m} \int_{K_{-}}^{K_{+}} \sqrt{K_{+}^{2}-q^{2}} q d q \\
& =\frac{1}{3} e \frac{\hbar}{(2 \pi)^{2} m}\left(K_{+}^{2}-K_{-}^{2}\right)^{3 / 2} .
\end{aligned}
$$

(c) $K_{+} \leqslant q$. All electrons are totally reflected, and there is no charge current flow, i.e., $J_{c}=0$.

Using the conventional definition of spin polarization

$$
P=\frac{n_{+}-n_{-}}{n_{+}+n_{-}}=\frac{K_{+}-K_{-}}{K_{+}+K_{-}},
$$

where $n_{ \pm}$are the majority or minority spin densities at Fermi level in the FMs, the ratio $J_{a} / J_{b}$ can be written as a function of polarization $P$ :

$$
\frac{J_{a}}{J_{b}}=\frac{(1+P)^{3}}{4 P^{3 / 2}}-2 .
$$

Note that $J_{b}$ is the maximal polarized current for the parallel FMs configuration. To get the total charge current, it should then be multiplied by the average transmission coefficient. Thus, the total electron current is given by ${ }^{9,30}$

$$
J_{e}=J_{a}+\left\langle T_{e}\right\rangle J_{b} .
$$

\section{B. Spin current and spin transfer torque}

The spin currents on two sides of $\mathrm{FM}_{B}$ are

$$
\begin{aligned}
& \mathbf{J}_{3 s}=\int d E \sum_{\mathbf{q}} \mathbf{j}_{3 s}(\mathbf{q}), \\
& \mathbf{J}_{4 s}=\int d E \sum_{\mathbf{q}} \mathbf{j}_{4 s}(\mathbf{q}),
\end{aligned}
$$

with spin current densities ${ }^{27}$

$$
\begin{aligned}
& \mathbf{j}_{3 s}(\mathbf{q})=\frac{\hbar^{2} k_{x}}{2 m}\left(\psi_{3, R}^{\dagger} \hat{\sigma} \psi_{3, R}-\psi_{3, L}^{\dagger} \hat{\sigma} \psi_{3, L}\right), \\
& \mathbf{j}_{4 s}(\mathbf{q})=\frac{\hbar^{2} k_{x}}{2 m}\left(\psi_{4, R}^{\dagger} \hat{\sigma} \psi_{4, R}-\psi_{4, L}^{\dagger} \hat{\sigma} \psi_{4, L}\right),
\end{aligned}
$$

where $\hat{\sigma}=\left(\sigma_{x}, \sigma_{y}, \sigma_{z}\right)$ are Pauli matrices. It is convenient to recast $\hat{\sigma}$ in local orthogonal coordinates of $\mathbf{m}_{B} \times\left(\mathbf{m}_{B} \times \mathbf{m}_{A}\right)$, $\mathbf{m}_{B} \times \mathbf{m}_{A}$, and $\mathbf{m}_{B}$ such that

$$
\hat{\sigma}=\sigma_{1} \mathbf{m}_{B} \times\left(\mathbf{m}_{B} \times \mathbf{m}_{A}\right)+\sigma_{2} \mathbf{m}_{B} \times \mathbf{m}_{A}+\sigma_{3} \mathbf{m}_{B},
$$

with

$$
\begin{gathered}
\sigma_{1}=\frac{1}{\sin \theta}\left(\begin{array}{cc}
-\sin \theta & \cos \theta e^{-i \phi} \\
\cos \theta e^{i \phi} & \sin \theta
\end{array}\right), \\
\sigma_{2}=\frac{1}{\sin \theta}\left(\begin{array}{cc}
0 & i e^{-i \phi} \\
-i e^{i \phi} & 0
\end{array}\right), \\
\sigma_{3}=\left(\begin{array}{cc}
\cos \theta & \sin \theta e^{-i \phi} \\
\sin \theta e^{i \phi} & -\cos \theta
\end{array}\right) .
\end{gathered}
$$

The STT on $\mathrm{FM}_{B}$ is equal to the difference of the spin currents on both sides of the ferromagnet, ${ }^{27,33}$

$$
\boldsymbol{\Gamma}=\mathbf{J}_{3 s}-\mathbf{J}_{4 s},
$$

and the STT density is

$$
\boldsymbol{\tau}=\mathbf{j}_{3 s}-\mathbf{j}_{4 s} .
$$

Thus, we have

$$
\boldsymbol{\tau}=a_{1} \mathbf{m}_{B} \times\left(\mathbf{m}_{B} \times \mathbf{m}_{A}\right)+a_{2} \mathbf{m}_{B} \times \mathbf{m}_{A}+a_{3} \mathbf{m}_{B},
$$


where

$$
\begin{aligned}
a_{i}= & \frac{\hbar^{2} k_{x}}{2 m}\left(\psi_{3, R}^{\dagger} \sigma_{i} \psi_{3, R}+\psi_{4, L}^{\dagger} \sigma_{i} \psi_{4, L}-\psi_{3, L}^{\dagger} \sigma_{i} \psi_{3, L}\right. \\
& \left.-\psi_{4, R}^{\dagger} \sigma_{i} \psi_{4, R}\right) \quad(i=1,2,3) .
\end{aligned}
$$

First of all, we can show $a_{3}=0$ because of the particle current conservation and the absence of spin-flipping. This can be understood as follows: We first rotate $\hat{z}$ to $\mathbf{m}_{B}$, then spinor state $\psi_{n, \alpha}=\hat{R}(\theta, \phi) \tilde{\psi}_{n, \alpha}$ where $\tilde{\psi}_{n, \alpha}$ is the electronic state seen along $\mathbf{m}_{B}$. Then, each spin density term $\psi_{n, \alpha}^{\dagger} \sigma_{3} \psi_{n, \alpha}$ in Eq. (33) becomes $\tilde{\psi}_{n, \alpha}^{\dagger} \hat{R}^{\dagger}(\theta, \phi) \sigma_{3} \hat{R}(\theta, \phi) \tilde{\psi}_{n, \alpha}=\tilde{\psi}_{n, \alpha}^{\dagger} \sigma_{z} \tilde{\psi}_{n, \alpha}$, so the majority spin state (parallel to $\mathbf{m}_{B}$,) and minority spin state (antiparallel to $\mathbf{m}_{B}$ ) are decoupled without mixing. In the absence of spin-flipping, both majority and minority electron currents are conserved. Thus, the STT projected along local magnetization $\mathbf{m}_{B}$ is zero. Here we have used the identity $\hat{R}^{\dagger}(\theta, \phi) \sigma_{3} \hat{R}(\theta, \phi)=\sigma_{z}$. The physical consequence is that STT can only rotate the magnetization without changing its magnitude.

Therefore, the STT can be divided into an in-plane (Slonczewski) term ${ }^{9} \boldsymbol{\tau}_{\|}=a_{1} \mathbf{m}_{B} \times\left(\mathbf{m}_{B} \times \mathbf{m}_{A}\right)$ and an outof-plane (fieldlike) term ${ }^{37} \boldsymbol{\tau}_{\perp}=a_{2} \mathbf{m}_{B} \times \mathbf{m}_{A}$. We note that the out-of-plane torque vanishes if $t_{u}, t_{d}, r_{u}$, and $r_{d}$ are real. This is because all the spins of both transmitted and reflected electrons are in the same plane spanned by $\mathbf{m}_{A}$ and $\mathbf{m}_{B}$ under the condition, and they can only vary in this plane.

Parameters $a_{1}$ and $a_{2}$ are important to understand the spin dynamics in a DSV. ${ }^{21,22}$ In Ref. 21 , these parameters are chosen to vary continuously without geometry dependence, while they are calculated in the diffusive transport limit in Ref. 22. To find $a_{1}$ and $a_{2}$ in our model, one needs to compute the quantities

$$
\begin{aligned}
T_{\sigma_{i}}\left(z_{1}\right)= & \frac{1}{2} \operatorname{Tr}\left(\hat{P}_{3, R}^{\dagger} \sigma_{i} \hat{P}_{3, R}-\hat{P}_{3, L}^{\dagger} \sigma_{i} \hat{P}_{3, L}-\hat{P}_{4, R}^{\dagger} \sigma_{i} \hat{P}_{4, R}\right. \\
& \left.+\hat{P}_{4, L}^{\dagger} \sigma_{i} \hat{P}_{4, L}\right) \quad(i=1,2),
\end{aligned}
$$

since $a_{i}=T_{\sigma_{i}}\left(z_{1}\right)\left(\hbar^{2} k_{x}\right) / m(i=1,2)$. Performing the same averaging procedure as we did on the charge current early, one finds

$$
\left\langle T_{\sigma_{i}}\right\rangle=\sum_{l_{i}=1}^{s_{i}} \operatorname{Res}\left(\frac{T_{\sigma_{i}}\left(z_{1}\right)}{z_{1}} ; z_{1, l_{i}}\right) \quad(i=1,2),
$$

where $z_{1, l_{i}}$ is the $l_{i}$ th pole of function $T_{\sigma_{i}}\left(z_{1}\right) / z_{1}$ inside the unit circle in the complex plane with $s_{i}$ the corresponding total pole number.

Since only region $b$ contributes to the spin current, ${ }^{9,30}$ one has $\left\langle a_{i}\right\rangle=\frac{\hbar}{e}\left\langle T_{\sigma_{i}}\right\rangle J_{b}(i=1,2)$. Thus, the average STT on the free magnetic layer is

$$
\boldsymbol{\Gamma}=g_{1}(\theta) \frac{\hbar}{e} J_{e} \mathbf{m}_{B} \times\left(\mathbf{m}_{B} \times \mathbf{m}_{A}\right)+g_{2}(\theta) \frac{\hbar}{e} J_{e} \mathbf{m}_{B} \times \mathbf{m}_{A},
$$

with scalar functions

$$
g_{i}(\theta)=\frac{\left\langle T_{\sigma_{i}}\right\rangle}{J_{a} / J_{b}+\left\langle T_{e}\right\rangle} \quad(i=1,2) .
$$

The STT $\boldsymbol{\Gamma}$ consists of two terms. The first one is Slonczewski torque, $\boldsymbol{\Gamma}_{\|}=g_{1}(\theta) \frac{\hbar}{e} J_{e} \mathbf{m}_{B} \times\left(\mathbf{m}_{B} \times \mathbf{m}_{A}\right)$, and the second one is fieldlike torque, $\boldsymbol{\Gamma}_{\perp}=g_{2}(\theta) \frac{\hbar}{e} J_{e} \mathbf{m}_{B} \times \mathbf{m}_{A}$.

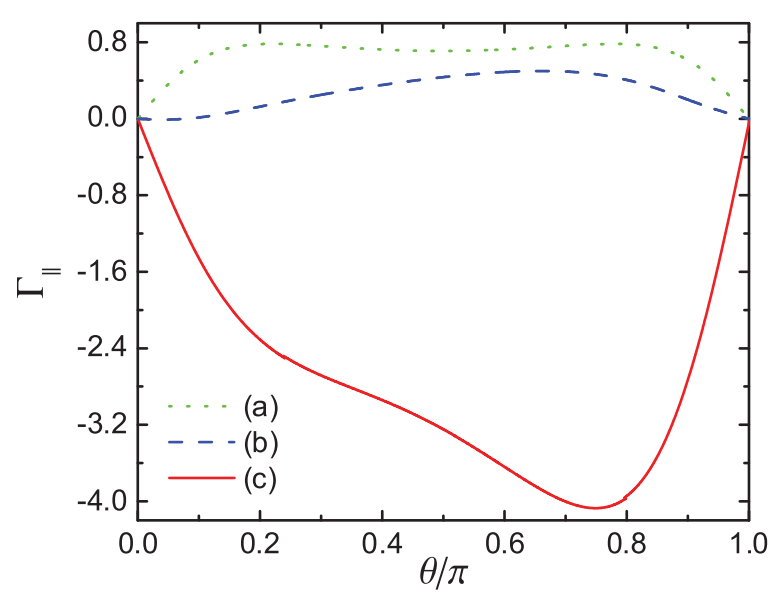

FIG. 3. (Color online) Slonczewski torque $\Gamma_{\|}$vs $\theta$ for polarization $P=0.6$ under three different conditions: (a) $t_{u}=1$ and $t_{d}=0$, (b) $\left|t_{u}\right|^{2}=0.99$ and $\left|t_{d}\right|^{2}=0.1$, and (c) $\left|t_{u}\right|^{2}=0.84$ and $\left|t_{d}\right|^{2}=0.17$. The unit of torque is $\frac{\hbar}{e} J_{e}$.

The general analytical forms of $g_{1}(\theta)$ and $g_{2}(\theta)$ are difficult to find because of the complicated residue calculations in Eqs. (16) and (35), except for a special case where $\mathrm{FM}_{B}$ is a perfect polarizer, which is demonstrated later. However, they can be numerically evaluated for any given material with definite material parameters $t_{u}, t_{d}, r_{u}$, and $r_{d}$. In Fig. 3, we present the numerical calculations of the magnitude of Slonczewski torque $\Gamma_{\|}=-g_{1}(\theta) \frac{\hbar}{e} J_{e} \sin \theta$ per unit current versus angle $\theta$ at polarization coefficient $P=0.6$ under three different conditions, which shows that different transmission probabilities have a strong impact on the torque. The STT is generally asymmetric against $\pi / 2$ for nonperfect polarizers. The torque found in the general case may become smaller than that in the ideal case, and it even changes its sign, relying on different transmission parameters. The reason is that, for nonperfect polarizers, both the majority and minority spins can transmit through the FM layer, which goes beyond the ideal case of Slonczewski's approximation of only allowing electrons either totally transmitted or reflected. ${ }^{9}$ A similar sign reversal of STT is demonstrated in the conventional spin valve. $^{31}$

Here, in order to directly compare our results in a DSV with Slonczewski's result in a conventional spin valve, ${ }^{9}$ let us consider the case of ideal $\mathrm{FM}_{B}$, i.e., $t_{u}=1$ and $t_{d}=0\left(r_{u}=\right.$ $\left.0, r_{d}=1\right)$. After some algebra, we obtain $T_{\sigma_{1}}=\frac{1}{4}\left(z_{1}+z_{1}^{*}-2\right)$ and $T_{\sigma_{2}}\left(z_{1}\right)=-\frac{i}{4}\left(z_{1}-z_{1}^{*}\right)$. The averaged values are then

$$
\begin{gathered}
\left\langle T_{\sigma_{1}}\right\rangle\left(t_{u}=1, t_{d}=0\right)=-\frac{1}{2}, \\
\left\langle T_{\sigma_{2}}\right\rangle\left(t_{u}=1, t_{d}=0\right)=0 .
\end{gathered}
$$

Thus, we get the scalar $g$ functions

$$
g_{1}(\theta)=\frac{-1}{-3+(1+P)^{3} /\left(2 P^{3 / 2}\right)-\left(\mathbf{m}_{A} \cdot \mathbf{m}_{B}\right)^{2}},
$$

$$
g_{2}(\theta)=0,
$$




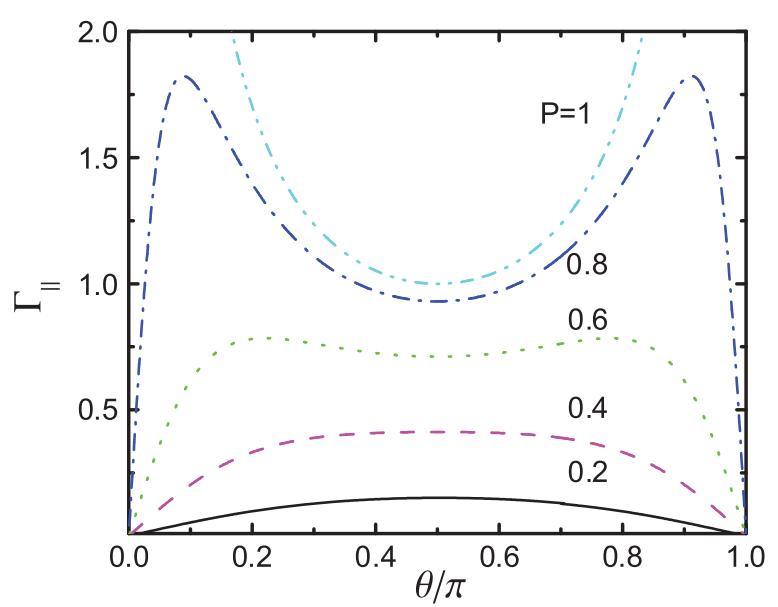

FIG. 4. (Color online) Slonczewski torque $\Gamma_{\|}$vs angle $\theta$ for various polarizations $P$ in the ideal case where all FMs are perfect polarizers. The unit of torque is $\frac{\hbar}{e} J_{e}$.

which is our main result and shows that only the Slonczewski torque exists in the ideal DSV. The absence of any layerthickness dependence in Eq. (40) results from the phase average across sufficiently thick normal metal layers. The values of $g_{1}(\theta=0, \pi)$ are crucial to evaluate the threshold current density $J_{e}^{*}$ needed for magnetization reversal of the free layer since $J_{e}^{*} \propto 1 / g_{1}(\theta=0, \pi){ }^{16}$

Figure 4 is the magnitude of STT per unit current as a function of angle $\theta$ for various polarization $P$ in an ideal DSV. The STT is symmetric against $\pi / 2$ due to the contributions from both fixed magnetic layers, which is different from the result in a conventional spin valve., ${ }^{9,30,31}$ For polarization coefficient $P<1$, it generally vanishes at $\theta=0$ and $\theta=\pi$ (shown in Fig. 4). However, if $P=1$, the torque is singular at $\theta=0$ and $\pi$. The divergence can be understood mathematically and from a physics viewpoint. Mathematically, the singularities at $\theta=0$ and $\pi$ are due to the factor of $g_{1}=-(\sin \theta)^{-2}$ when $P=1$. Physically this is the consequence of a perfect spin filter. In our model, every electron transfers its angular momentum to the local magnetization when it impinges the interface of a magnetic layer whose magnetization is not parallel to its spin. ${ }^{9}$ Meanwhile, the STT per unit current is defined as the spin transfer per transmitted electron. ${ }^{9}$ However, in the case of $\theta=0$ or $\theta=\pi$, perfect spin filter does not allow any electron transmission through the DSV, which leads to a zero electron transmission and results in the divergence. The above argument can also be applied to the STT divergence at $\theta=\pi$ in a traditional spin valve in the original paper by Slonczewski. ${ }^{9}$ Nevertheless, we find that the total STT, $\Gamma=-\frac{1}{2} \frac{\hbar}{e} J_{b} \mathbf{m}_{B} \times\left(\mathbf{m}_{B} \times \mathbf{m}_{A}\right)$ if $P=1$, does not have such singularities.

Finally, we would like to compare the magnitude of STT in our DSV with that obtained in a traditional spin valve. ${ }^{9}$ In Slonczewski's original paper, ${ }^{9}$ he calculated the $g$ function in spin valve,

$$
g_{1}^{*}(\theta)=\frac{-1}{-4+(1+P)^{3}\left(3+\mathbf{m}_{A} \cdot \mathbf{m}_{B}\right) /\left(4 P^{3 / 2}\right)},
$$

which was later reproduced by Krstajić et al. in Ref. 30 .

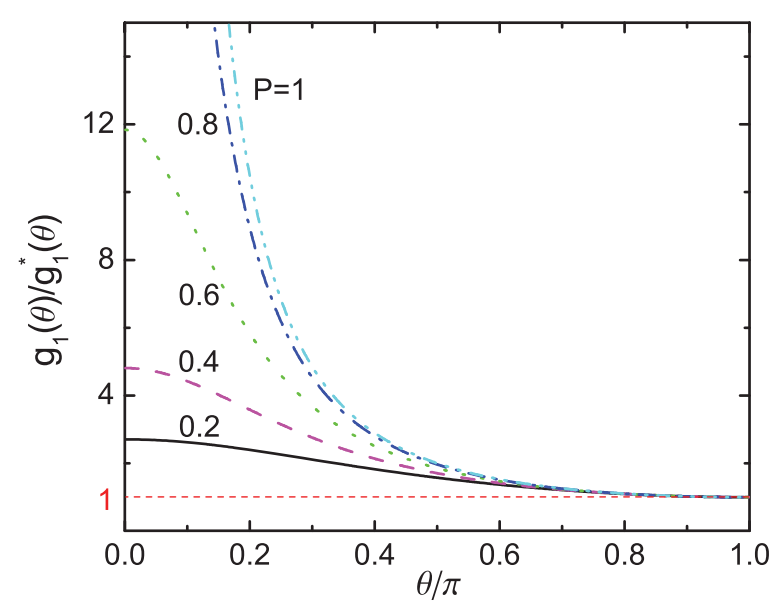

FIG. 5. (Color online) Spin transfer torque enhancement ratio $g_{1}(\theta) / g_{1}^{*}(\theta)$ vs $\theta$ for various polarizations $P$ in the ideal case where all FMs are perfect polarizers.

We plot the ratio $g_{1}(\theta) / g_{1}^{*}(\theta)$ as a function of angle $\theta$ for various polarization $P$ in Fig. 5. One can see that the STT is largely enhanced when $\theta$ is acute, but it approaches that in a conventional spin valve when $\theta>\pi / 2$. Namely, the enhancement value sensitively depends on both the orientation of the free layer and the polarization degree of the electrons. Under small tilted angle $\theta$ and large polarization $P$, the enhancement ratio is dramatic, which is consistent with Berger's predictions ${ }^{20}$ and substantially decreases the required threshold current density and the switching time for magnetization reversal. For instance, $g_{1} / g_{1}^{*}=11.9$ if $\theta=\frac{\pi}{6}$ and $P=0.8$, while the enhancement ratio approaches 1 (red dashed line shown in Fig. 5) when $\theta$ is close to $\pi$. The reason is that there is no difference between a DSV and a conventional spin valve when layers $\mathrm{FM}_{A}$ and $\mathrm{FM}_{B}$ are aligned in an antiparallel manner, since $\mathrm{FM}_{C}$ would not reflect electrons coming out of $\mathrm{FM}_{B}$ in such a case. We can see that the torque enhancement totally comes from the contribution of the additional ferromagnetic layer $\mathrm{FM}_{C}$ in comparison with Slonczewski's result. ${ }^{9}$ These results are qualitatively consistent with Fuchs et al.'s experiment, ${ }^{38}$ which shows that the reduction of threshold current switching $\mathrm{FM}_{B}$ from parallel to antiparallel, with respect to $\mathrm{FM}_{A}$, is substantial, whereas it is only of modest size from antiparallel to parallel in a DSV compared to that in a conventional spin valve.

From the qualitative physical picture and quantummechanical calculations, we conclude that STT can be greatly enhanced in a DSV compared to that in a spin valve structure in the ballistic regime. The findings of the physics behind and the analytical formula of STT in this emerging geometry should be interesting for both theoretical ${ }^{21,22}$ and experimental ${ }^{38,39}$ concerns. Micromagnetic simulation based on our new $g$ function [Eq. (40)] and the Landau-Lifshitz-Gilbert equation ${ }^{40}$ is a direction of future research. The behavior of STT in an asymmetric DSV when the widths of two NMs are different (i.e., $l_{A} \neq l_{B}$ ) is also an interesting issue for further investigation.

Although the advantage of STT enhancement is unambiguously demonstrated in our results, the accuracy of the analytical formula still needs experimental confirmation. We 
suggest the use of a recently developed technique called spin-transfer-driven ferromagnetic resonance ${ }^{41}$ to measure the angular dependence of the STT in a DSV.

\section{SUMMARY}

In conclusion, we derive the STT acting on the free magnetic layer in a DSV structure in the ballistic regime. A quantum-mechanical description of the STT is presented, which is valid for nanoscale DSVs where both the electron mean free path and the spin-flip relaxation length are larger than the spacer thickness. ${ }^{23}$ Using a quasi-one-dimensional model and within the Slonczewski approach, we obtained the analytical form of the STT when all magnetic layers are perfect polarizers. Similar to the results in the diffusive regime, the STT is dramatically enhanced in comparison to that in a conventional spin valve in the ballistic regime. Depending on the orientation of the free magnetic layer and the polarization degree of the electrons, the STT can be enhanced by a factor of a few tens. Our analytical $g$ function provides a theoretical base for the micromagnetic simulation of the spin dynamics in DSV. The general cases when transmission and reflection probabilities of the free layer are different from zero or one are also numerically calculated, which shows that the torque may be lowered and may change its sign under different transmission probabilities. These results should be useful for switching current reduction in magnetization reversal.

\section{ACKNOWLEDGMENTS}

This work is supported by Hong Kong RGC (Grants No. 603508, No. 604109, No. RPC10SC05, and No. HKU10/CRF/08-HKUST17/CRF/08). X.R.W. would like to acknowledge the hospitality of the Kavli Institute for Theoretical Physics China, CAS. Z.Z.S. thanks the Alexander von Humboldt Foundation (Germany) for a grant.

\section{APPENDIX: MATRIX EXPRESSION}

After some algebra, the matrices in Eqs. (9) and (10) are

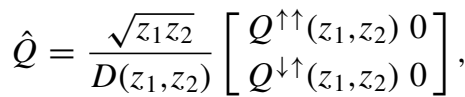

$$
\begin{aligned}
& \hat{P}_{3, R}=\frac{\sqrt{z_{1}}}{D\left(z_{1}, z_{2}\right)}\left[\begin{array}{c}
P_{3, R}^{\uparrow \uparrow}\left(z_{1}, z_{2}\right) \\
P_{3, R}^{\downarrow \uparrow}\left(z_{1}, z_{2}\right)
\end{array}\right] \text {, }
\end{aligned}
$$

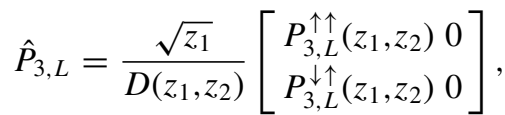

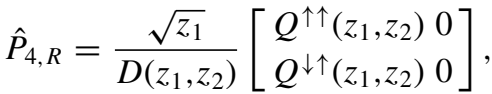

$$
\begin{aligned}
& \hat{P}_{4, L}=\frac{z_{2} \sqrt{z_{1}}}{D\left(z_{1}, z_{2}\right)}\left[\begin{array}{cc}
Q^{\uparrow \uparrow}\left(z_{1}, z_{2}\right) & 0 \\
0 & 0
\end{array}\right],
\end{aligned}
$$

where $z_{1}=\exp \left(i 2 \varphi_{A}\right), z_{2}=\exp \left(i 2 \varphi_{B}\right)$, and

$$
\begin{aligned}
& Q^{\uparrow \uparrow}\left(z_{1}, z_{2}\right)=t_{u} \cos ^{2} \frac{\theta}{2}+t_{d} \sin ^{2} \frac{\theta}{2} \\
& -z_{1}\left(r_{d} t_{u} \cos ^{2} \frac{\theta}{2}+r_{u} t_{d} \sin ^{2} \frac{\theta}{2}\right), \\
& Q^{\downarrow \uparrow}\left(z_{1}, z_{2}\right)=\frac{1}{2} e^{i \phi} \sin \theta\left[t_{u}-t_{d}\right. \\
& +\left(z_{1}+z_{2}\right)\left(r_{u} t_{d}-r_{d} t_{u}\right) \\
& \left.+z_{1} z_{2}\left(t_{u} r_{d}^{2}+t_{d} t_{u}^{2}-t_{u} t_{d}^{2}-t_{d} r_{u}^{2}\right)\right], \\
& D\left(z_{1}, z_{2}\right)=1-z_{1}\left(r_{u} \sin ^{2} \frac{\theta}{2}+r_{d} \cos ^{2} \frac{\theta}{2}\right) \\
& -z_{2}\left(r_{u} \cos ^{2} \frac{\theta}{2}+r_{d} \sin ^{2} \frac{\theta}{2}\right) \\
& +z_{1} z_{2}\left[r_{u} r_{d}+\frac{\sin ^{2} \theta}{4}\left(\left(r_{u}-r_{d}\right)^{2}-\left(t_{u}-t_{d}\right)^{2}\right)\right], \\
& P_{3, R}^{\uparrow \uparrow}\left(z_{1}, z_{2}\right)=D\left(z_{1}, z_{2}\right), \\
& P_{3, R}^{\downarrow \uparrow}\left(z_{1}, z_{2}\right)=\frac{1}{2} z_{1} e^{i \phi} \sin \theta\left[r_{u}-r_{d}\right. \\
& +z_{2}\left(-r_{u}^{2}+r_{u} r_{d}+t_{u}^{2}-t_{u} t_{d}\right) \cos ^{2} \frac{\theta}{2} \\
& \left.+z_{2}\left(r_{d}^{2}-r_{u} r_{d}-t_{d}^{2}+t_{u} t_{d}\right) \sin ^{2} \frac{\theta}{2}\right], \\
& P_{3, L}^{\uparrow \uparrow}\left(z_{1}, z_{2}\right)=z_{2}\left(t_{u} \cos ^{2} \frac{\theta}{2}+t_{d} \sin ^{2} \frac{\theta}{2}\right)^{2} \\
& -z_{2}\left(r_{u} \cos ^{2} \frac{\theta}{2}+r_{d} \sin ^{2} \frac{\theta}{2}\right)^{2}+r_{u} \cos ^{2} \frac{\theta}{2} \\
& +r_{d} \sin ^{2} \frac{\theta}{2}+z_{1} z_{2}\left[r_{u}\left(r_{d}^{2}-t_{d}^{2}\right) \sin ^{2} \frac{\theta}{2}\right. \\
& \left.+r_{d}\left(r_{u}^{2}-t_{u}^{2}\right) \cos ^{2} \frac{\theta}{2}\right] \text {, } \\
& P_{3, L}^{\downarrow \uparrow}\left(z_{1}, z_{2}\right)=\frac{P_{3, R}^{\downarrow \uparrow}\left(z_{1}, z_{2}\right)}{z_{1}} .
\end{aligned}
$$

The notation $X^{\downarrow \uparrow}\left(X=\hat{Q}, \hat{P}_{3, R}, \hat{P}_{3, L}\right)$ refers to the transition amplitude from spin-up to spin-down states.
${ }^{1}$ J. A. Katine, F. J. Albert, R. A. Buhrman, E. B. Myers, and D. C. Ralph, Phys. Rev. Lett. 84, 3149 (2000).

${ }^{2}$ J. Grollier, V. Cros, A. Hamzic, J. M. George, H. Jaffrès, A. Fert, G. Faini, J. B. Youssef, and H. Legall, Appl. Phys. Lett. 78, 3663 (2001).
${ }^{3}$ K. Xia, P. J. Kelly, G. E. W. Bauer, A. Brataas, and I. Turek, Phys. Rev. B 65, 220401(R) (2002).

${ }^{4}$ S. Urazhdin, N. O. Birge, W. P. Pratt, and J. Bass, Phys. Rev. Lett. 91, 146803 (2003).

${ }^{5}$ M. Tsoi, J. Z. Sun, and S. S. P. Parkin, Phys. Rev. Lett. 93, 036602 (2004). 
${ }^{6}$ T. Y. Chen, S. X. Huang, C. L. Chien, and M. D. Stiles, Phys. Rev. Lett. 96, 207203 (2006).

${ }^{7}$ Y. Zhou, C. L. Zha, S. Bonetti, J. Persson, and J. Åkerman, Appl. Phys. Lett. 92, 262508 (2008).

${ }^{8}$ X. Chen, Q. R. Zheng, and G. Su, Phys. Rev. B 78, 104410 (2008).

${ }^{9}$ J. C. Slonczewski, J. Magn. Magn. Mater. 159, L1 (1996).

${ }^{10}$ L. Berger, Phys. Rev. B 54, 9353 (1996).

${ }^{11}$ Z. Z. Sun and X. R. Wang, Phys. Rev. B 71, 174430 (2005); 73, 092416 (2006); 74, 132401 (2006).

${ }^{12}$ X. R. Wang, P. Yan, J. Lu, and C. He, Ann. Phys. (NY) 324, 1815 (2009); X. R. Wang, P. Yan, and J. Lu, Europhys. Lett. 86, 67001 (2009).

${ }^{13}$ A. Yamaguchi, T. Ono, S. Nasu, K. Miyake, K. Mibu, and T. Shinjo, Phys. Rev. Lett. 92, 077205 (2004).

${ }^{14}$ M. Hayashi, L. Thomas, C. Rettner, R. Moriya, Y. B. Bazaliy, and S. S. P. Parkin, Phys. Rev. Lett. 98, 037204 (2007).

${ }^{15}$ P. Yan and X. R. Wang, Phys. Rev. B 80, 214426 (2009); Appl. Phys. Lett. 96, 162506 (2010); P. Yan, Z. Z. Sun, J. Schliemann, and X. R. Wang, Europhys. Lett. 92, 27004 (2010).

${ }^{16}$ X. R. Wang and Z. Z. Sun, Phys. Rev. Lett. 98, 077201 (2007); X. R. Wang, P. Yan, J. Lu, and C. He, Europhys. Lett. 84, 27008 (2008).

${ }^{17}$ H. Z. Lu and S. Q. Shen, Phys. Rev. B 80, 094401 (2009).

${ }^{18}$ M. Hatami, G. E. W. Bauer, Q. Zhang, and P. J. Kelly, Phys. Rev. Lett. 99, 066603 (2007).

${ }^{19}$ Z. Yuan, S. Wang, and K. Xia, Solid State Commun. 150, 548 (2010).

${ }^{20}$ L. Berger, J. Appl. Phys. 93, 7693 (2003).

${ }^{21}$ C. Y. You, J. Appl. Phys. 107, 073911 (2010).

${ }^{22}$ P. Baláž, M. Gmitra, and J. Barnaś, Phys. Rev. B 80, 174404 (2009).

${ }^{23}$ F. J. Jedema, M. S. Nijboer, A. T. Filip, and B. J. van Wees, Phys. Rev. B 67, 085319 (2003); J. Barnaś, A. Fert, M. Gmitra, I. Weymann, and V. K. Dugaev, ibid. 72, 024426 (2005).
${ }^{24}$ T. Valet and A. Fert, Phys. Rev. B 48, 7099 (1993).

${ }^{25}$ J. Xiao, A. Zangwill, and M. D. Stiles, Phys. Rev. B 70, 172405 (2004).

${ }^{26}$ C. Heiliger, M. Czerner, B. Y. Yavorsky, I. Mertig, and M. D. Stiles, J. Appl. Phys. 103, 07A709 (2008).

${ }^{27}$ M. D. Stiles and A. Zangwill, Phys. Rev. B 66, 014407 (2002).

${ }^{28}$ D. Waldron, P. Haney, B. Larade, A. MacDonald, and H. Guo, Phys. Rev. Lett. 96, 166804 (2006).

${ }^{29}$ A. Brataas, Y. V. Nazarov, and G. E. W. Bauer, Phys. Rev. Lett. 84, 2481 (2000); Eur. Phys. J. B 22, 99 (2001).

${ }^{30}$ P. M. Krstajić, M. Keller, and F. M. Peeters, Phys. Rev. B 77, 174428 (2008).

${ }^{31}$ X. Waintal, E. B. Myers, P. W. Brouwer, and D. C. Ralph, Phys. Rev. B 62, 12317 (2000).

${ }^{32}$ J. Xiao, G. E. W. Bauer, and A. Brataas, Phys. Rev. B 77, 224419 (2008).

${ }^{33}$ F. Romeo and R. Citro, Phys. Rev. B 81, 045307 (2010).

${ }^{34} \mathrm{~S}$. Datta, Electronic Transport in Mesoscopic Conductors (Cambridge University, New York, 1995).

${ }^{35}$ M. Gmitra and J. Barnaś, Appl. Phys. Lett. 89, 223121 (2006).

${ }^{36}$ M. D. Stiles, J. Appl. Phys. 79, 5805 (1996).

${ }^{37}$ S. Zhang, P. M. Levy, and A. Fert, Phys. Rev. Lett. 88, 236601 (2002).

${ }^{38}$ G. D. Fuchs, I. N. Krivorotov, P. M. Braganca, N. C. Emley, A. G. F. Garcia, D. C. Ralph, and R. A. Buhrman, Appl. Phys. Lett. 86, 152509 (2005).

${ }^{39}$ M. Watanabe, J. Okabayashi, H. Toyao, T. Yamaguchi, and J. Yoshino, Appl. Phys. Lett. 92, 082506 (2008).

${ }^{40}$ T. L. Gilbert, IEEE Trans. Magn. 40, 3443 (2004).

${ }^{41}$ J. C. Sankey, P. M. Braganca, A. G. F. Garcia, I. N. Krivorotov, R. A. Buhrman, and D. C. Ralph, Phys. Rev. Lett. 96, 227601 (2006); J. C. Sankey, Y. T. Cui, J. Z. Sun, J. C. Slonczewski, R. A. Buhrman, and D. C. Ralph, Nat. Phys. 4, 67 (2008). 